\title{
Effort Control and Judgments
}

\author{
J. Frank Yates and Roy M. Kulick
}

The University of Michigan

\begin{abstract}
This study examined the interrelationships among judgments of task effort, performance, and difficulty. Sixty subjects provided judgments in the form of ratings of 28 hypothetical tasks in which they might have engaged. Performance level judgments were expressed as subjective probabilities of success at the tasks. The subjective relationship between effort and performance at a task was characterized by the way such probabilities of success changed as a function of effort devoted to the task. Contrary to the hypothesis suggested by the inverted-U Yerkes-Dodson (1908) relationship, subjects generally believed performance level to increase monotonically with effort. Subjects' perceived effort-performance relationships were examined for two characteristics: (a) the maximum achievable levels of performance and (b) the degree to which performance level was seen as a positively or negatively accelerated function of effort. It is argued that these two characteristics of effort-performance functions capture important and distinct aspects of task difficulty. Indexes of these characteristics derived from subjective effort-performance functions jointly accounted for $60 \%$ of the variance in overall task difficulty ratings. Implications of the approach and results of the study for effort control and current models of motivation are discussed.
\end{abstract}

Why do we devote more effort to one activity than to another? The flow diagram model of Fig. 1 suggests a useful way of thinking about this focal motivation problem. The model, similar to that proposed by Kahneman (1973), distinguishes two interrelated systems that are responsible for action, a "performance system" and an "effort control system." Productivity at a task is construed as the material output of the person's performance system, comprised of his mental and physical characteristics. The functioning of that performance system in turn depends on several inputs. First, it is limited by the availability of external resources that might be applied to the task at hand, e.g., tools and energy sources. The quality of performance also depends on the nature of the task itself. Performance at one task might be poorer than performance at another because the tasks require operations differing in complexity. Finally, performance level depends on the extent to which available resources are actually applied to

We express our thanks to Glenda Vogt and Carolyn Jagacinski for their computerprogramming assistance with the data analyses. We are also indebted to David $\mathrm{H}$. Krantz and Ms. Jagacinski for their helpful criticisms and suggestions. Requests for reprints should be sent to J. Frank Yates, Department of Psychology, 136 Perry Building, University of Michigan, Ann Arbor, Michigan 48109. 


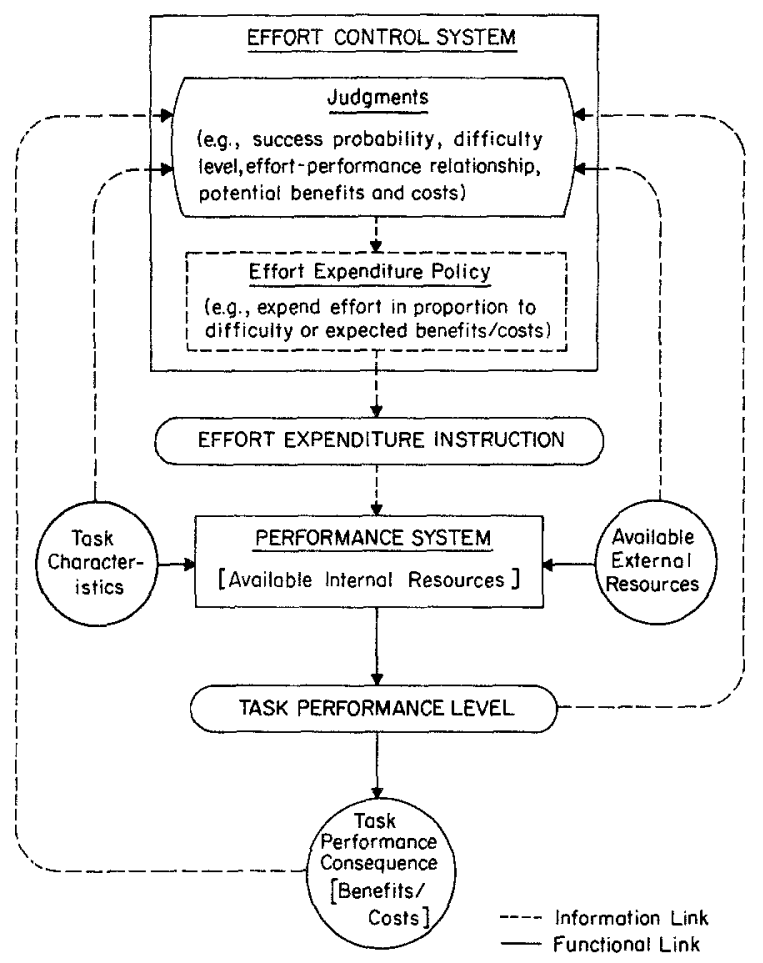

FIG. 1. Flow diagram model of effort and performance.

the task, in a word, "effort" (cf. Kahneman, 1973; Pribram \& McGuinness, 1975). By available resources we mean not only resources external to the person, but resources internal to the performance system itself, e.g., mental capacity and metabolic energy sources. The performance system expends effort according to instructions from the effort control system. The object of the present research is to shed some light on the role of judgments in the operation of the effort control system.

The effort control system transmits instructions according to some policy. An effort expenditure policy amounts to a set of rules stating that when certain conditions exist, then certain instructions are appropriate. Theories of motivation may be viewed as claims about the nature of effort expenditure policies. Most current theories of human motivation emphasize the role of judgments in effort control. Within the framework of the proposed model, such judgments may be seen as reports of some of the conditions to which an effort expenditure policy is applied. Some of those reports or perceptions concern environmental circumstances, e.g., task characteristics, available external resources, and performance consequences. Others are equivalent to feedback to the effort control system 
about the functioning of the performance system, e.g., how productivity responds to various levels of effort expended.

Which judgments are used by the effort control system and what is their status in the expenditure policy? Subjective probabilities of successful performance and anticipated performance consequences are the critical judgments according to expectancy theories, a class of motivation models enjoying a great deal of current popularity (Atkinson, 1964; Vroom, 1964; Lawler, 1973). These models assert that task effort should depend on one's opinion of the extent to which one's effort will produce good performance, which, in turn, will lead to desirable consequences. Although the details of various expectancy theory formulations differ, they all share the claim of a multiplicative tradeoff between success probabilities and the value of performance consequences. In other words, as long as a minimal chance of successful performance exists, the inclination of a person to expend effort at a task can be increased by enhancing the attractiveness of the consequences of successful performance, and vice versa.

Kahneman and his associates (Kahneman, 1973; Kahneman, Peavler, \& Onuska, 1968) approach the problem of effort control from a different perspective, that of capacity models of attention. On the basis of experimental and anecdotal evidence, Kahneman makes the strong statement that task difficulty is the overriding determinant of effort allocation, far outstripping the influence of performance consequences and intentions. Implicitly, this assertion is a claim for the primacy of difficulty judgments in effort expenditure policies. This follows, since only a judgment of task difficulty is generally available at the time a task is undertaken, not perfectly reliable knowledge of the actual difficulty level.

Any type of effort expenditure policy must certainly involve the most fundamental judgment of all, a perception of the relationship between expended effort and resulting performance level. Evidence from a variety of sources suggests that the true relationship is that of an inverted- $U$, the so-called Yerkes and Dodson (1908) function (Anderson, 1976; Kahneman, 1973, pp. 33-37; Näätämen, 1973; Vroom, 1964, pp. 204-209). But how do people ordinarily believe performance responds to effort? If the judged relationship is discrepant from the true relationship, then misallocations of effort resulting in poor performance are inevitable. Moreover, as will be explained, if perceived effort-performance relationships have certain characteristics, then it should be possible to account for specific instances of overallocation or underallocation of effort.

The research reported here is an initial attempt to coordinate the roles played in effort control by the three classes of judgments described above, success probabilities, difficulty assessments, and perceptions of effort-performance relationships. While a great deal of past research in motivation and decision theory has addressed probability and difficulty 
judgments, there has been little attention directed toward summarizing generally perceived effort-performance relationship judgments. And certainly there has been no examination of connections among the judgments. Nevertheless, it seems that an effort control system would demand that certain interrelationships hold. Intuition suggests specific minimal requirements: For instance, one should have low probabilities of success at difficult tasks. It should also be necessary to invest more effort in a difficult task than in an easy one in order to perform well. As we will show, the actual interrelationships among these various judgments are necessarily richer and more complex than these intuitions suggest.

To facilitate an examination and possible integration of the judgments in question, we required subjects to report their opinions in a purposefully constrained fashion. Specifically, the subject considered each of a series of common tasks in which performance was categorized dichotomously as success or failure. For each task, the subject indicated his subjective probabilities of success $(P(S \mid E))$ conditional on various levels of (subjective) effort he might devote to the task. Within this framework, performance level was defined as the magnitude of the probability of successful task performance. Besides their subjective success probabilities, subjects were also asked to disclose their judgments of the difficulty levels for the same tasks. The subject reported these judgments as ratings of the difficulty of each task for him personally.

\section{METHOD}

The subjects were recruited from the University of Michigan Human Performance Center's paid subject pool. The group of 60 subjects was evenly divided between males and females. The average age of the subjects was 21.4 years. Each subject was paid $\$ 2.00$ for participation in the study. While subjects worked in groups of 5-10 persons, each subject reported his individual judgments in the form of responses to a private questionnaire.

The questionnaire administered to subjects contained two parts, one for eliciting task difficulty opinions and the other for eliciting judgments of the relationship between effort and performance. Alternative forms of the questionnaire, each presented to half the subject sample, counterbalanced the order of presentation of the two parts. The questionnaire contained brief descriptions of 28 different tasks. Figure 2 contains a listing of four representative tasks employed.

The questionnaire did not require subjects to report judgments about tasks whose natures they did not understand. Following each description the subject indicated his judgment of the task's difficulty via a continuous scale with anchors labeled "Not At All Difficult" and "Extremely Difficult." The subject was requested to make a slash through the scale at the point "corresponding to how difficult you believe the task to be for you." 
Type A: Skilled Physical Task

Attain a score of 140 or above in a bowling game.

Type B: Unskilled Physical Task

Sit alone on a chair in an otherwise empty room. Stay awake for 36 hours.

Type C: Longterm Mental Task

Attain a grade of $A$ in organic chemistry.

Type D: Shortterm Mental Task

Complete the following word gome. Find 15 or more words of four or more letters in the word "ENHANCED" (There exist more than 15.) Time limit: 20 minutes.

Fig. 2. Descriptions of representative tasks considered by subjects.

For purposes of eliciting effort-performance relationship judgments, the subject was asked to imagine the range of effort he could conceivably devote to the task to be partitioned by seven equally spaced points. The terminal points were labeled "No Effort" and "Greatest Possible Effort." For each task, the subject was asked to rate what his chances of success at the task would be, given that he devoted the amount of effort represented by each of the seven levels partitioning the effort continuum. The subject reported these seven opinions as numbers on a 100-point scale where 0 indicated "no chance of succeeding" and 100 corresponded to a feeling of being "absolutely certain" of succeeding.

\section{RESULTS AND DISCUSSION \\ Effort-Performance Relationship}

Subjective probabilities of successful performance at various effort levels $(P(S \mid E))$ were indexed by the subject's ratings on the 100-point chance of success scales. The primary concern in the present context is the shape of the graph of success probability as a function of effort. Figure $3 \mathrm{~A}$ presents examples of idealized graphs consistent with the Yerkes-Dodson relationship. Of the entire group of 60 subjects, the responses of only two subjects consistently indicated that such graphs represented their beliefs about the relationship between effort and chances of success. The remaining subjects overwhelmingly reported a monotone increasing relationship between effort expenditure and subjective probability of success. So, regardless of the true relationship between effort and performance, it appears that subjects generally believe that increased effort results in increased chances of successful performance.

Exactly how subjects' perceptions of the effort-performance relationship affect effort allocation depends on the effort expenditure policy. That policy might easily include tradeoffs between the benefits of devoting 
more effort and the costs of doing so, e.g., fatigue. Nevertheless, if performance is indeed determined by effort in the fashion suggested by the Yerkes-Dodson relationship, oversufficient allocations of effort should not be particularly rare occurrences. There should be common instances in which people invest a lot of effort in a task and tend to perform much worse than they anticipate.

$P(S \mid E)$ curves, such as those illustrated in Fig. 3, can have countless different shapes and still be monotone increasing in subjective effort. Such distinctions in curve shapes may be significant in their implications for actual effort allocations. Accordingly, the $P(S \mid E)$ curves derived for subjects were examined for certain distinguishing features. Sometimes the curves were slightly sigmoid in shape. However, the most important distinctions among the monotone curves are illustrated in Fig. 3B. First, compare Curves $a$ and $b$. The primary difference between the curves is that their maximum points are not the same. From the subject's point of view, under the best conditions for the respective tasks, his chances of success at Task a are better than those at Task $b$. In this special sense, it is reasonable to say that Task a is thought to be less "difficult" than Task b. In abbreviated form we might define "Difficulty 1"' for a task as $\Delta_{1}=1$ $P(S \mid E)_{\max }$, the complement of the maximum value assumed by $P(S \mid E)$.

Consider Curves b, $c$, and d. All three tasks are equally difficult in the sense of Difficulty 1. However, Difficulty 1 does not likely tell the whole story about how judgments of the corresponding tasks influence allocations of effort to them. Their clearly different contours have plausible interpretations and may have practical implications, depending on effort expenditure policies. While the person producing the curves is indicated to believe his chances of success are the same for all three tasks when he (subjectively) expends no effort or the greatest possible effort, what hap-
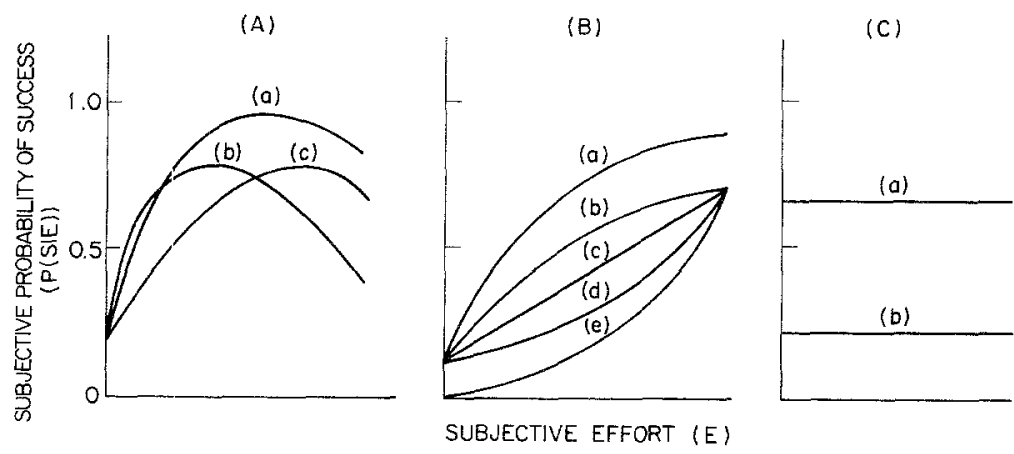

FIG. 3. Hypothetical subjective probability of success functions of effort: (A) Yerkes-Dodson relationship curves; (B) major types of monotone increasing curves; (C) nonresponsive curves differing in elevation. 
pens between those extremes is another matter. One interpretation of the distinctions concerns the probability of success at each intermediate level of effort expenditure. In the sense that the chances of success at each intermediate effort level are less for Task $d$ than for Tasks $b$ and $c$, it is reasonable to say that Task $d$ is more difficult than either of the others in a way distinct from Difficulty 1 . Similarly, Task $\mathrm{c}$ is more difficult than Task $b$ in this alternative sense of the term. For convenience, call this second form of difficulty, "Difficulty 2."

Another interpretation of the differences among Curves $b, c$, and $d$ is also possible. It concerns the rate at which $P(S \mid E)$ increases as a function of subjective effort. Curve $c$ suggests that the person conceives of a constant moderate rate of increase in $P(S \mid E)$ with subjective effort for Task $c$. On the other hand, Curve $b$ implies that success at Task $b$ is very responsive to increases in subjective effort when one is starting from no effort. However, that responsiveness diminishes increasingly. In contrast, success at Task $d$ is very unresponsive to effort increases from the no effort level, but becomes very responsive as one approaches the maximum effort expenditure. It seems that whether a $P(S \mid E)$ curve is concave like Curve b (low in Difficulty 2) or convex like Curve d (high in Difficulty 2) might have consequences for actual effort allocations. Suppose the effort expenditure policy is one that takes into account a tradeoff between the increase in $P(S \mid E)$ as effort increases and the costs associated with such effort increments. A concave, negatively accelerated, $P(S \mid E)$ curve would incline the person to be very willing to devote at least a minimal amount of effort to the relevant task. However, the person should not be expected to expend large amounts of effort since he does not believe such great effort is needed to achieve a reasonably high probability of success. In contrast, if a person believes the $P(S \mid E)$ curve for a task to be convex, or positively accelerated, then allocations of minimal amounts of effort should be rare. When any effort is devoted at all, it should be high because the person believes high effort to be necessary to produce even a modest chance of success at the task.

There is an additional possible distinction among $P(S \mid E)$ curves that should be noted. Compare Curve e to all the others. The important difference is that Curve e has a minimum $P(S \mid E)$ of 0 . Given that one of the effort levels subjects were to bring to mind was "No Effort," all curves should have $P(S \mid E)_{\min }=0$. This was not always indicated to be so by the data. Apparently, many subjects did not interpret "No Effort" literally but, rather, thought of it as something of a lower threshold of effort.

The fact that subjects did not always believe $P(S \mid E)_{\min }=0$ is related to another observed phenomenon that is somewhat surprising, the occurrence of horizontal $P(S \mid E)$ curves as illustrated in Fig. 3C. The curves suggest that the person believes performance at either Task $a$ or Task $b$ is 
completely unresponsive to effort. What is curious about such curves is that they are not always horizontal at $P(S \mid E)=0$. In other words, although effort changes beyond the threshold level are not perceived to influence performance, the threshold level of effort itself is thought to be capable of producing performance at distinct, non-negligible levels for various tasks.

What types of $P(S \mid E)$ curves were, in fact, indicated by subjects? To simplify the coding of curves, the following analytical procedures were employed. As suggested above, Difficulty 1 was indexed by $\Delta_{1}=1-$ $P(S \mid E)_{\max }$. Difficulty 2 is a harder concept to summarize. To arrive at a somewhat adequate index we assumed that $P(S \mid E)$ could be represented as a power function of subjective effort: $P(S \mid E)=\alpha E^{\theta}$. Within limits, the exponent in such an expression can serve as an index of the convexity or concavity of the corresponding curve. To gain an intuitive grasp of this fact, observe that when $\theta=1$, the curve is linear; when $\theta=1 / 2$, the curve is half of a parabola oriented horizontally; and when $\theta=2$, the curve is half of a parabola oriented vertically. So, we set our Difficulty 2 index as $\Delta_{2}=\theta$ for the power function that best fits the $P(S \mid E)$ ratings provided by a given subject for a given task. The exact procedure for estimating $\Delta_{2}$ required two operations. First, to eliminate all values of $P(S \mid E)=0$, one point was added to each success probability $P(S \mid E)$ ) rating. The net effect of this is to vertically translate each $P(S \mid E)$ curve slightly. The second operation was taking logarithms of both sides of $P(S \mid E)=\alpha E^{\Delta 2}$, where $E$ was set at the integer values $1-7$. Note that $\log P(S \mid E)=\log \left(\alpha E^{\Delta 2}\right)=\log \alpha$ $+\Delta_{2} \log E . \Delta_{2}$ was approximated as the linear regression coefficient for predicting $\log P(S \mid E)$ from $\log E$. While the translation of the curves results in a slight distortion of this estimate from the true exponent value, such coefficients should adequately serve as indicators of the basic meaning of the Difficulty 2 concept.

In general, the level of linear predictability of $\log P(S \mid E)$ by $\log E$ was excellent. The responses of the subject with the worst linear fits resulted in a mean $r$ across tasks of .85. The highest across-task mean $r$ for an individual subject was .98 , while the average mean $r$ across all subjects was .93 .

It appears that, at least for the 28 tasks used in this study, convex $P(S \mid E)$ curves predominate. Over all 58 subjects with primarily monotone $P(S \mid E)$ curves and over all tasks, the mean proportion of $\Delta_{2}$ values greater than 1.0 was .60. Another view of the $\Delta_{2}$ indexes confirms the prevalence of convex curves. For 24 subjects, the occurrence of concave and convex curves was significantly different from what would be expected under the assumption that such curve types are equally likely $\left(p<.05, \chi^{2}(1)\right)$. Only five of those subjects evidenced more concave than convex $P(S \mid E)$ curves. Although horizontal curves were not ubiquitous, they were by no 
means rare. Over the 28 tasks employed in this study, the mean number of horizontal $P(S \mid E)$ curves was 1.93 for the 58 subjects who did not report that performance level eventually declines with effort. In summary, it seems that while subjects generally believe chances of success to become more favorable with increases in expended effort, they also tend to expect the advantages of such increased effort to accelerate as the total amount of effort becomes higher. Nevertheless, they sometimes anticipate that effort expenditure has no effect on their performance at all.

An attempt was made to see if different types of tasks tended to induce $P(S \mid E)$ curves that were characteristically convex or concave. Accordingly, a panel of 15 experimental psychology professors and graduate students were asked to place each of the 28 tasks of the present study into one of the four task categories illustrated in Fig. 2: Type A, Skilled Physical Task; Type B, Unskilled Physical Task; Type C, Long-Term Mental Task; and Type D, Short-Term Mental Task. There were, respectively, nine, four, five, and ten tasks placed in the categories. For each subject, the proportions of tasks for which he reported convex $P(S \mid E)$ curves within each category were computed. Such proportions provided an index of the extent to which tasks of the respective types had characteristic $P(S \mid E)$ curve shapes. The incidence of convex $P(S \mid E)$ curves was greatest for the unskilled physical tasks and least for the short-term mental tasks. That is, it appeared that subjects were inclined to believe that effort was increasingly beneficial when applied to unskilled physical tasks, but not so for other kinds of tasks. A general conclusion of this type must await further evidence, however. An analysis of variance applied to transformed proportions of positively accelerated $P(S \mid E)$ curves within task categories indicated only marginally significant task type differences $(F(3,171)=2.49, .05<p<.10)$.

A similar analysis was performed to see whether different types of tasks varied in their tendencies to induce horizontal $P(S \mid E)$ curves. Again, for each subject the proportions of tasks for which he reported horizontal $P(S \mid E)$ curves within each task category were computed. An analysis of variance applied to variance-stabilizing transforms of those proportions suggested that the incidence of horizontal $P(S \mid E)$ curves does indeed depend on task type $(F(3,171)=3.95, p<.01)$. An examination of contrasts among the means of the transformed proportions revealed the nature of that dependency. The incidence of horizontal $P(S \mid E)$ curves was significantly lower for skilled physical tasks than for the other three types of tasks $(t=-3.16, p<.05$, Scheffé comparison). In other words, in comparison to unskilled physical tasks and mental tasks (whether longterm or short-term), subjects were much less inclined to believe that effort expended on a skilled physical task would prove to be futile. 


\section{Subjective Difficulty Judgments}

What about the relationship between subjective probabilities of success and judgments of task difficulty? As mentioned earlier, intuition suggests that tasks judged to be difficult should elicit low subjective probabilities of success. Since success or failure at a task depends on effort, however, such a general statement is not very meaningful. Probabilities of success must be conditionalized on expended effort. Accordingly, our approach to the problem of relating task difficulty and success probability judgments was to regress task difficulty ratings on Difficulty 1 and Difficulty 2 indexes derived from corresponding $P(S \mid E)$ curves. The results of such a procedure might suggest the extent to which generalized task difficulty opinions are influenced by perceptions of the phenomena we have called Difficulty 1 and Difficulty 2 , i.e., maximum achievable performance level and the extent to which performance level is a positively accelerated function of effort expenditure.

For 57 of the 58 subjects evidencing generally monotone $P(S \mid E)$ curves, multiple $R$ was statistically significant $(p<.05)$; for the remaining case, $p=$ .06 . The mean value of $R^{2}$ across all subjects was .6003 . That is, on the average $60 \%$ of the variance in difficulty ratings could be explained by Difficulty $1\left(\Delta_{1}\right)$ and Difficulty $2\left(\Delta_{2}\right)$ indexes. In 55 of 58 cases $\Delta_{1}$ provided significant unique contributions to $R^{2}(p<.05) ; \Delta_{2}$ provided similar contributions for 31 of the 58 subjects with monotone $P(S \mid E)$ curves. Over all 58 cases, the mean increment in $R^{2}$ provided by adding $\Delta_{1}$ to $\Delta_{2}$ as a predictor of difficulty ratings was .4122 , while the corresponding mean increment was .0962 for $\Delta_{2}$. In summary, then, it appears that difficulty judgments are systematically related to subjective probabilities of success. Although such judgments seem to be influenced primarily by the greatest possible success probability the subject can project, they also tend to be affected by changes in the rate at which success probabilities respond to effort expenditure.

\section{GENERAL DISCUSSION}

The results of the present study not only lead to specific suggestions about effort control mechanisms, but offer some potentially useful ways of viewing several other psychological phenomena as well. Consider first the use in this study of probabilities of success conditional on effort expended $(P(S \mid E))$. Curiously, the explicit use of such conditional probabilities rather than unconditional probabilities of success represents a departure from most motivational analyses reported to date (Atkinson, 1964; Weiner, 1972; Korman, 1974). Kukla's (1972) statement of expectancy theory is one of the few treatments in the motivation literature incorporating the concept of conditionalized success probabilities. Without question, the most well- 
known version of expectancy theory among personality psychologists is Atkinson's (1964) theory of achievement motivation. That model asserts that one's "resultant tendency" to undertake a risky task is given by $T=$ $\left(M_{\mathrm{S}}-M_{\mathrm{AF}}\right) P_{\mathrm{S}}\left(1-P_{\mathrm{S}}\right)$, where $M_{\mathrm{S}}$ and $M_{\mathrm{AF}}$ are, respectively, indexes of the person's motives to succeed and avoid failure and $P_{\mathrm{S}}$ is his subjective probability of success at the task. Regardless of the data base supporting or challenging this model, a moment's reflection should highlight its conceptual difficulties. The model is clearly incomplete in that an unconditional probability of success is very ambiguous. One's probability of success at most tasks depends on how much effort one devotes to them. The results of the present study clearly demonstrate that people generally believe this to be so. A more adequate version of Atkinson's achievement motivation model must certainly take into account the dependency of subjective probabilities of success on intended effort.

Weiner $(1972,1974)$ and his associates have been most responsible for the application of attribution theoretical concepts to motivation problems. The Weiner models highlight the motivational significance of attributions of performance level to such factors as ability, task difficulty, effort, and luck. While the evidence gathered in support of their claims is broad, the formulation and analytical approaches of the Weiner group are lacking in clarity. For instance, their discussions leave the impression that ability, task difficulty, effort, and luck are seen by subjects as independent and distinct contributors to performance. This hardly seems reasonable, but the studies to date have offered no straightforward way of examining the perceived relationships among such causal factors. Perhaps $P(S \mid E)$ curve analyses of the type used in the present study provide such an approach. It would be useful to pursue such plausible hunches such as that performance at tasks with horizontal $P(S \mid E)$ curves is seen to be strongly influenced by luck or, when a $P(S \mid E)$ curve is very steep, success would be attributed to effort. One result of this study suggests the potential fruitfulness of a $P(S \mid E)$ curve approach. One of the tasks considered by the subjects was predicting the number of games the Detroit Tigers baseball team would win during the upcoming season. It seems plausible that many people would think that one's accuracy at making such a prediction is largely dependent on luck or chance, i.e., factors one can neither know nor control. This task was, in fact, one of those with the highest incidences of horizontal $P(S \mid E)$ curves.

Finally, the distinction made in the present study between Difficulty 1 and Difficulty 2 suggests a corresponding distinction between two types of ability. At least conceptually, one can envision constructing $P(S \mid E)$ curves in which the probabilities are actuarial or "objective" rather than subjective. Assuming that the true relationship between effort and performance conforms to the Yerkes-Dodson function, curves such as those in Fig. 3A 
would be representative. One type of ability at a task, "Ability 1 ," might be indexed by the maximum point of the corresponding $P(S \mid E)$ curve. Ability 1 represents something like a limit on one's performance at a task. As suggested by Curves $\mathrm{b}$ and $\mathrm{c}$ in Fig. 3A, one may have equal Ability 1 at two tasks, yet still differ in ability for the tasks in another way. While Curves $b$ and $c$ have peaks at the same level, the curves reach their peaks at different rates. Maximum performance level for Task $b$ is reached with a lower effort expenditure than for Task $\mathrm{c}$. We might say the person is "quicker" at Task b than Task c; his Ability 2 level is greater for Task b than for Task c. It is important to recognize that, for a given task, Ability 1 and Ability 2 may or may not covary across individuals. Identification of these two distinct kinds of capacity might form the basis for more effective personnel and guidance policies than exist presently. Current hiring and advising procedures based heavily on paper-and-pencil testing techniques seem to reflect Ability 2 almost exclusively.

\section{REFERENCES}

Anderson, C. R. Coping behaviors as intervening mechanisms in the inverted-U stressperformance relationship. Journal of Applied Psychology, 1976, 61, 30-34.

Atkinson, J. W. An introduction to motivation. New York: Van Nostrand Reinhold, 1964.

Kahneman, D. Attention and effort. Englewood Cliffs, New Jersey: Prentice-Hall, 1973.

Kahneman, D., Peavler, W. S., \& Onuska, L. Effects of verbalization and incentive on the pupil response to mental activity. Canadian Journal of Psychology, 1968, 22, 186-196.

Korman, A. K. The psychology of motivation. Englewood Cliffs, New Jersey: Prentice-Hall, 1974.

Kukla, A. Foundations of an attributional theory of performance. Psychological Review, $1972,79,454-470$.

Lawler, E. E. Motivation in work organizations. Monterey, California: Brooks-Cole, 1973.

Näätänen, $R$. The inverted-U relationship between activation and performance: A critical review. In S. Kornblum (Ed.), Attention and performance IV. New York: Academic Press, 1973.

Pribram, K. H., \& McGuinness, D. Arousal, activation, and effort in the control of attention. Psychological Review, 1975, 82, 116-149.

Vroom, V. H. Work and motivation. New York: Wiley, 1964.

Weiner, B. Theories of motivation. Chicago: Markham, 1972.

Weiner, B. (Ed.) Achievement motivation and attribution theory. Morristown, New Jersey: General Learning Press, 1974.

Yerkes, R. M., \& Dodson, J. D. The relation of strength of stimulus to rapidity of habit formation. Journal of Comparative and Neurological Psychology, 1908, 18, 459-482.

Recerved: January 3, 1977 